\title{
Risk of left atrial appendage thrombus in patients with atrial fibrillation and chronic kidney disease
}

\author{
Monika Budnik ${ }^{1}$, Monika Gawałko ${ }^{1}$, Iwona Gorczyca ${ }^{2,3}$, Beata Uziębło-Życzkowska ${ }^{4}$, \\ Paweł Krzesiński ${ }^{4}$, Janusz Kochanowski ${ }^{1}$, Piotr Scisło ${ }^{1}$, Anna Michalska ${ }^{3}$, \\ Olga Jelonek ${ }^{2}$, Katarzyna Starzyk ${ }^{2,3}$, Agnieszka Jurek ${ }^{4}$, Marek Kiliszek $^{4}$, \\ Beata Wożakowska-Kapłon²,3, Grzegorz Gielerak ${ }^{4}$, Krzysztof J. Filipiak ${ }^{1}$, \\ Grzegorz Opolski ${ }^{1}$, Agnieszka Kapłon-Cieślicka ${ }^{1}$
}

${ }^{1} 1^{\text {st }}$ Chair and Department of Cardiology, Medical University of Warsaw, Poland

${ }^{2} 1^{\text {st }}$ Clinic of Cardiology and Electrotherapy, Swietokrzyskie Cardiology Center, Kielce, Poland

${ }^{3}$ Collegium Medicum, The Jan Kochanowski University, Kielce, Poland

${ }^{4}$ Department of Cardiology and Internal Diseases, Military Institute of Medicine, Poland

\begin{abstract}
Background: Atrial fibrillation ( $A F$ ) and chronic kidney disease (CKD) are associated with an increased risk of ischemic stroke. The aim of this study was to compare the clinical characteristics, the incidence of left atrial appendage (LAA) thrombus and its predictors, and spontaneous echo contrast (SEC) in a population of patients with $A F$ depending on estimated glomerular filtration rate (eGFR) values.

Methods: This study included 1962 patients who underwent transesophageal echocardiographic examination prior to cardioversion or ablation in the years 2014-2018 in three cardiac centers.

Results: More than a quarter of AF patients had decreased eGFR $\left(<60 \mathrm{~mL} / \mathrm{min} / 1.73 \mathrm{~m}^{2}\right)$ and were characterized as a high-risk population, with more comorbidities, higher thromboembolic and bleeding risk compared to those with normal renal function. Oral anticoagulation (OAC) was prescribed in 97\% and $93 \%$ of patients with decreased and normal eGFR, respectively, with a higher prevalence of prescribed non-vitamin $K$ antagonist oral anticoagulants (NOACs). The incidence of LAA thrombus $(24 \%, 9 \%$ and $4 \%)$ and SEC $(25 \%, 25 \%$ and $19 \%)$ increases simultaneously with a decrease in eGFR $\left(<30,30-59\right.$ and $\geq 60 \mathrm{~mL} / \mathrm{min} / 1.73 \mathrm{~m}^{2}$, respectively). Among patients prescribed reduced doses of NOAC, those with decreased eGFR were more often observed with LAA thrombus (10\% vs. 2.5\%). Non-paroxysmal $A F$, heart failure and previous bleeding were predictors of LAA thrombus, irrespective of eGFR value. CKD was the predictor of LAA thrombus in all patients including those with non-paroxysmal $A F$, males, without diabetes, without hypertension and with $\mathrm{CHA}_{2} D S_{2}-V A S c<2$.

Conclusions: Despite OAC, patients with concomitant $A F$ and CKD remain at high risk for $L A A$ thrombus formation. (Cardiol J 2022; 29, 2: 205-215)
\end{abstract}

Key words: oral anticoagulation, renal failure, stroke prevention, thromboembolic risk

\section{Introduction}

Atrial fibrillation (AF) occurs in approximately $3 \%$ adults aged 20 years or older with a greater prevalence in the elderly and patients with greater comorbid burden [1, 2]. It is an important risk factor for ischemic stroke since it associates with a 5 -fold higher risk of stroke compared with the general population [3]. Tromboembolic events were identified in about $12 \%$ of cases for AF patients [4]. An-

Address for correspondence: Iwona Gorczyca, $\mathrm{MD}, \mathrm{PhD}, 1^{\text {st }}$ Clinic of Cardiology and Electrotherapy, Swietokrzyskie Cardiology Center, ul. Grunwaldzka 45, 25-736 Kielce, Poland, tel: +48 41367 15 10, fax: +48 413671396 , e-mail: iwona.gorczyca@interia.pl

Received: 01.01.2020 Accepted: 24.01.2020 Early publication date: 18.03.2020

This article is available in open access under Creative Common Attribution-Non-Commercial-No Derivatives 4.0 International (CC BY-NC-ND 4.0) license, allowing to download articles and share them with others as long as they credit the authors and the publisher, but without permission to change them in any way or use them commercially. 
ticoagulation treatment with vitamin $\mathrm{K}$ antagonists (VKAs) or non-VKA oral anticoagulants (NOACs), reducing the incidence of stroke and mortality [5]. Chronic kidney disease (CKD) alone is associated with a higher incidence of both strokes and bleeding $[6,7]$. Moreover, patients with advanced CKD were excluded in large clinical trials (trials with dabigatran, rivaroxaban, and edoxaban excluded patients with an estimated glomerular filtration rate $(\mathrm{eGFR})<30 \mathrm{~mL} / \mathrm{min} / 1.73 \mathrm{~m}^{2}$, with apixaban - those with eGFR were $<25 \mathrm{~mL} / \mathrm{min} / 1.73 \mathrm{~m}^{2}$ or creatinine $>2.5 \mathrm{mg} / \mathrm{dL}$ ) [8-11]. Kidney function should be regularly monitored in AF patients on oral anticoagulants (OACs) to allow dose adaptation and to estimate risk of complications [12].

Previous studies suggest that the incidence of left atrial appendage (LAA) thrombi or spontaneous echo contrast (SEC) is up to $29 \%$ [13].

The aim of the present study was to compare the incidence of the LAA thrombi and SEC in a population of patients with $\mathrm{AF}$ who underwent transesophageal echocardiographic (TEE) examination prior to cardioversion or ablation depending on eGFR, a comparison of clinical characteristic of patients according to eGFR and determination of risk factors of LAA thrombi.

\section{Methods}

\section{Study population}

The study included consecutive patients with $\mathrm{AF}$ undergoing TEE before cardioversion or ablation between 2014 and 2018 from three large cardiac centers in Poland (an academic, military and district hospitals). In the academic department, TEE was performed routinely in all patients regardless of the duration of $\mathrm{AF}$ and the anticoagulant therapy. In the military and district hospitals, TEE was performed in cases when there was doubt regarding the timing of anticoagulant treatment and patient compliance. All TEE studies were performed by certified echocardiographers (certified with accreditation of the Section of Echocardiography of the Polish Cardiac Society), using EPIQ 7 Ultrasound Machine $^{\circledR}$ (Philips Medical Systems, Andover, Massachusetts, United States), iE33 Ultrasound Machine $^{\circledR}$ (Philips Medical Systems), General Electric Vivid 7 (GE Healthcare, Milwaukee, Wisconsin, United States) or E95 Ultrasound Machine ${ }^{\circledR}$ (GE Healthcare). Written informed consent for TEE was obtained from all patients.

Data on the clinical characteristics of patients, echocardiographic findings, laboratory results were retrospectively retrieved from patients' medi- cal history. The study protocol was approved by the Bioethics Committee. In addition, due to the retrospective nature of the study and the lack of additional interventions, the Committee waived the requirement to obtain separate consent from each patient to participate in the study.

The current analysis included only patients with data on baseline eGFR. This was estimated based on creatinine measurement at hospital admission (i.e. before TEE), using the Modification of Diet in Renal Disease (MDRD) Study equation. Patients were divided into three groups according to eGFR $(<30 \mathrm{~mL} /$ $/ \mathrm{min} / 1.73 \mathrm{~m}^{2}-21$ patients, $30-59 \mathrm{~mL} / \mathrm{min} / 1.73 \mathrm{~m}^{2}$ -509 patients and $\geq 60 \mathrm{~mL} / \mathrm{min} / 1.73 \mathrm{~m}^{2}-1432 \mathrm{pa}-$ tients). However, due to the small number of patients in the group with eGFR $<30 \mathrm{~mL} / \mathrm{min} / 1.73 \mathrm{~m}^{2}$, the multivariate logistic regression analysis of the two groups are included (eGFR $\geq 60 \mathrm{~mL} / \mathrm{min} / 1.73 \mathrm{~m}^{2}$ and $<60 \mathrm{~mL} / \mathrm{min} / 1.73 \mathrm{~m}^{2}$ ).

\section{Statistical analysis}

Data is presented as a median and interquartile range (IQR) or number of patients and percentages where appropriate. The statistical significance of differences in medians was analyzed using the Kruskal-Wallis test. Frequencies of parameters or events were compared using the $\chi^{2}$ test or the Fisher exacttest, as appropriate. Forall tests, ap value $<0.05$ was considered to be statistically significant. To determine predictors of LAA thrombus formation, univariate and multivariate logistic regression analyses were performed. Only variables that were available for more than $88 \%$ of patients were included in the logistic regression analysis. Statistical analysis was performed with StatsModels: Statistic in Python — v0.10.1 documentation.

\section{Results}

\section{Basic characteristic}

Compared to other groups, patients with eGFR $<60 \mathrm{~mL} / \mathrm{min} / 1.73 \mathrm{~m}^{2}$ were older, more often suffered from hypertension, diabetes, coronary artery disease, peripheral vascular disease, heart failure, cancer and more often were female. Moreover, the incidence of previous thromboembolic complications definied as composite of stroke/ /transient ischemic attack and/or peripheral embolism as well as hemorrhagic events were higher in patients with the lowest value of eGFR. Detailed clinical characteristics of the groups are presented in Table 1. 
Non-vitamin K antagonist oral anticoagulants were least often prescribed in the group with eGFR $<30 \mathrm{~mL} / \mathrm{min} / 1.73 \mathrm{~m}^{2}$ with a higher prevalance of low-dose NOAC prescription. There were no significant differences in the frequency of antiplatelet therapy or bridging therapy with heparin among all eGFR groups.

\section{Comparison of LAA thrombi prevalence}

The incidence of LAA thrombi was more than twice as high in patients with eGFR $30-60 \mathrm{~mL} /$ $/ \mathrm{min} / 1.73 \mathrm{~m}^{2}$ than in those with $\geq 60 \mathrm{~mL} / \mathrm{min} / 1.73 \mathrm{~m}^{2}$ (9\% vs. $4 \%$, respectively). In a relatively small group of patients with eGFR $<30 \mathrm{~mL} / \mathrm{min} / 1.73 \mathrm{~m}^{2}$ thrombi were present in almost a quarter of patients (24\%). In addition, the lowest LAA emptying velocity were observed in the group of patients with eGFR $<30 \mathrm{~mL} / \mathrm{min} / 1.73 \mathrm{~m}^{2}$ (31 vs. 42 vs. $50 \mathrm{~cm} / \mathrm{s}$, respectively). Detailed therapeutic characteristics and echocardiographic findings of the eGFR groups are presented in Table 2.

\section{Predictors of LAA thrombus}

On multivariate logistic regression, for the whole study group, eGFR was one of the predictors of LAA thrombus $(\mathrm{p}=0.04)$. The other predictors were age, non-paroxysmal AF, heart failure, previous bleeding (Table 3 ), similar to patients with eGFR $\geq 60 \mathrm{~mL} / \mathrm{min} / 1.73 \mathrm{~m}^{2}$ (Table $4 \mathrm{~A}$ ). In those with eGFR $<60 \mathrm{~mL} / \mathrm{min} / 1.73 \mathrm{~m}^{2}$, non-paroxysmal $\mathrm{AF}$, hypertension, heart failure and previous bleeding were the predictors of LAA thrombus (Table 4B).

Chronic kidney disease was the predictor of LAA thrombus in all patients as well as in those with non-paroxysmal AF (but not with paroxysmal AF), in males (but not in females), without diabetes, without hypertension and with $\mathrm{CHA}_{2} \mathrm{DS}_{2}-\mathrm{VASc}<2$ i.e. groups not included in classic risk factors (Fig. 1). Among patients with LAA thrombus no differences retaled to OAC treatment were observed between patients with $\mathrm{eGFR}<60 \mathrm{~mL} / \mathrm{min} / 1.73 \mathrm{~m}^{2}$ and eGFR $\geq 60 \mathrm{~mL} / \mathrm{min} / 1.73 \mathrm{~m}^{2}$ (Table 5). Among patients on reduced dose of NOAC, LAA thrombus occured more often in patients with eGFR $<60$ $\mathrm{mL} / \mathrm{min} / 1.73 \mathrm{~m}^{2}$ than in those with eGFR $\geq 60 \mathrm{~mL} /$ $/ \mathrm{min} / 1.73 \mathrm{~m}^{2}$ (Table 6 ).

\section{Comparison of patients without OAC}

Analyzing patients who were not treated with OAC (neither VKA nor NOAC) (Table 7), the incidence of LAA thrombus was higher and LAA emptying velocity was lower in patients with eGFR $<60 \mathrm{~mL} / \mathrm{min} / 1.73 \mathrm{~m}^{2}$ than in those with eGFR $<$ $60 \mathrm{~mL} / \mathrm{min} / 1.73 \mathrm{~m}^{2}$. Patients with eGFR $<60 \mathrm{~mL} /$
$/ \mathrm{min} / 1.73 \mathrm{~m}^{2}$ more often had persistent $\mathrm{AF}$ and heart failure as compared to those with normal eGFR. Median $\mathrm{CHA}_{2} \mathrm{DS}_{2}$-VASc score was 2 in patients with eGFR $<60 \mathrm{~mL} / \mathrm{min} / 1.73 \mathrm{~m}^{2}$ and 1 in patients with eGFR $\geq 60 \mathrm{~mL} / \mathrm{min} / 1.73 \mathrm{~m}^{2}$. More than $50 \%$ of patients in both groups were found to be at high thromboembolic risk $\left(\mathrm{CHA}_{2} \mathrm{DS}_{2}\right.$-VASc score $\left.\geq 2\right)$.

\section{Discussion}

The major findings of the present study are as follows. First, more than a quarter of $\mathrm{AF}$ patients had decreased eGFR $\left(<60 \mathrm{~mL} / \mathrm{min} / 1.73 \mathrm{~m}^{2}\right)$ and simultaneously were characterized as a highrisk population, with more comorbidities, higher thromboembolic and bleeding risk compared to those with normal renal function. Second, OAC was prescribed in approximately $97 \%$ of patients with decreased eGFR ( $90.5 \%$ of patients with eGFR $<30 \mathrm{~mL} / \mathrm{min} / 1.73 \mathrm{~m}^{2}$ ). The higher prevalence of prescribed NOAC was observed among patients with eGFR $30-59 \mathrm{~mL} / \mathrm{min} / 1.73 \mathrm{~m}^{2}$. Importantly, among patients prescribed with reduced doses of NOAC, those with eGFR $<60 \mathrm{~mL} / \mathrm{min} / 1.73 \mathrm{~m}^{2}$ were more often observed with LAA thrombus. Third, the most important finding was that CKD was the predictor of LAA thrombus in all patients as well as in the group that are not included in classic risk factors.

Numerous observational studies yielded conflicting results for $\mathrm{OAC}$ regarding which of the two types of anticoagulant drug, NOAC vs. VKA, is preferable for patients with decreased eGFR. In the present study, among patients with LAA thrombus, there was no difference reflected to OAC treatment between patients with eGFR $<60$ and $\geq 60 \mathrm{~mL} / \mathrm{min} / 1.73 \mathrm{~m}^{2}$. It is in line with a previous study [14] which proved that none of the $\mathrm{OAC}$ regimens predicted LAA thrombus in patients with AF, as well as with other studies focused on thromboembolic risk among AF patients with CKD [15-17]. Pivotal randomized controlled trials have established that NOAC are superior, however without statistical significance, to VKA among patients with CKD in preventing thromboembolic events. The ROCKET AF study indicates that, when compared with warfarin, rivaroxaban was non-inferior in preventing stroke or systemic embolism. Among patients with creatinine clearance $30-49 \mathrm{~mL} / \mathrm{min}$, the primary endpoint of stroke or systemic embolism occurred in 2.32 per 100 patient-years with rivaroxaban $15 \mathrm{mg}$ /day vs. 2.77 per 100 patientyears with warfarin, whereas among those with creatinine clearance $\geq 50 \mathrm{~mL} / \mathrm{min}$, the primary end- 
Table 1. Clinical characteristics in all groups according to eGFR.

\begin{tabular}{|c|c|c|c|c|}
\hline Variable & $\begin{array}{c}\text { Patients with } \\
\text { eGFR }<30 \\
\text { (n = 21) }\end{array}$ & $\begin{array}{l}\text { Patients with } \\
\text { eGFR 30-59 } \\
\text { (n = 509) }\end{array}$ & $\begin{array}{l}\text { Patients with } \\
\text { eGFR } \geq 60 \\
\text { (n }=1432 \text { ) }\end{array}$ & $\mathbf{P}$ \\
\hline Age [years] & $71[60-82]$ & $67[62-72]$ & $61[54-67]$ & $<0.0001$ \\
\hline Female & $9(43 \%)$ & $281(55 \%)$ & $424(30 \%)$ & $<0.0001$ \\
\hline $\mathrm{BMI}\left[\mathrm{kg} / \mathrm{m}^{2}\right]$ & $\begin{array}{c}26[24-30] \\
n=14\end{array}$ & $\begin{array}{c}29[26-32] \\
n=347\end{array}$ & $\begin{array}{c}29 \text { [26-32]; } \\
\mathrm{n}=1158\end{array}$ & 0.34 \\
\hline Obesity & $\begin{array}{c}4(22 \%) \\
n=17\end{array}$ & $\begin{array}{c}145(39 \%) \\
n=370\end{array}$ & $\begin{array}{c}483(40 \%) \\
n=1204\end{array}$ & 0.49 \\
\hline \multicolumn{5}{|l|}{ Type of AF } \\
\hline Paroxysmal AF & $6(29 \%)$ & $197(39 \%)$ & $740(52 \%)$ & $<0.0001$ \\
\hline Persistent AF & $11(52 \%)$ & $279(55 \%)$ & $622(43 \%)$ & $<0.0001$ \\
\hline Permanent/long-standing persistent AF & $4(19 \%)$ & $33(6.5 \%)$ & $70(4.9 \%)$ & 0.02 \\
\hline \multicolumn{5}{|l|}{ Concomitant diseases } \\
\hline Hypertension & $17(81 \%)$ & $395(78 \%)$ & $965(67 \%)$ & $<0.0001$ \\
\hline Dyslipidemia & $5(24 \%)$ & $180(35 \%)$ & $532(37 \%)$ & 0.57 \\
\hline Diabetes & $6(29 \%)$ & $114(22 \%)$ & $243(17 \%)$ & 0.01 \\
\hline CAD & $6(29 \%)$ & $114(22 \%)$ & $240(17 \%)$ & 0.01 \\
\hline Previous MI & $5(24 \%)$ & $53(10 \%)$ & $88(6.1 \%)$ & $<0.0001$ \\
\hline Previous $\mathrm{PCl} / \mathrm{CABG}$ & $5(24 \%)$ & $55(11 \%)$ & $104(7.3 \%)$ & 0.002 \\
\hline PAD & $\begin{array}{c}2(11 \%) \\
n=18\end{array}$ & $\begin{array}{c}14(3.4 \%) \\
n=407\end{array}$ & $\begin{array}{c}29(2.8 \%) \\
\mathrm{n}=1031\end{array}$ & 0.11 \\
\hline Vascular disease (CAD and/or PAD) & $7(33 \%)$ & $126(25 \%)$ & $263(18 \%)$ & 0.004 \\
\hline Heart failure & $9(43 \%)$ & $147(29 \%)$ & $250(18 \%)$ & $<0.0001$ \\
\hline Previous stroke/TIA/peripheral embolism & $3(14 \%)$ & $49(9.6 \%)$ & $85(5.9 \%)$ & 0.02 \\
\hline Chronic respiratory disease & $\begin{array}{c}1(5.6 \%) \\
n=18\end{array}$ & $\begin{array}{c}32(7.9 \%) \\
n=406\end{array}$ & $\begin{array}{c}54(5.2 \%) \\
\mathrm{n}=1031\end{array}$ & 0.15 \\
\hline Liver disease & $\begin{array}{c}1(5.6 \%) \\
n=18\end{array}$ & $\begin{array}{c}2(0.5 \%) \\
n=407\end{array}$ & $\begin{array}{c}19(1.8 \%) \\
\mathrm{n}=1031\end{array}$ & 0.06 \\
\hline Malignancy & $\begin{array}{c}1(6.7 \%) \\
n=15\end{array}$ & $\begin{array}{c}27(8.7 \%) \\
n=309\end{array}$ & $\begin{array}{c}39(4.5 \%) \\
n=869\end{array}$ & 0.018 \\
\hline Previous bleeding & $4(19 \%)$ & $41(8.1 \%)$ & $56(3.9 \%)$ & $<0.0001$ \\
\hline Labile INR & $\begin{array}{l}0(0 \%) \\
\mathrm{n}=18\end{array}$ & $\begin{array}{c}9(2.2 \%) \\
\mathrm{n}=407\end{array}$ & $\begin{array}{l}9(0.9 \%) \\
\mathrm{n}=1031\end{array}$ & 0.10 \\
\hline Smoking & $\begin{array}{c}2(13 \%) \\
n=15\end{array}$ & $\begin{array}{c}95(31 \%) \\
n=309\end{array}$ & $\begin{array}{c}282(33 \%) \\
n=869\end{array}$ & 0.30 \\
\hline \multicolumn{5}{|l|}{ Thromboembolic risk } \\
\hline $\mathrm{CHADS}_{2}$ score & $\begin{array}{c}2.2 \pm 1.3 \\
2[1-3]\end{array}$ & $\begin{array}{c}1.6 \pm 1.2 \\
1[1-2]\end{array}$ & $\begin{array}{c}1.2 \pm 1.0 \\
1[0-2]\end{array}$ & $<0.0001$ \\
\hline $\mathrm{CHA}_{2} \mathrm{DS}_{2}$-VASc score & $\begin{array}{c}3.7 \pm 2.0 \\
3[3-5]\end{array}$ & $\begin{array}{c}3.1 \pm 1.7 \\
3[2-4]\end{array}$ & $\begin{array}{c}2.0 \pm 1.5 \\
2[1-3]\end{array}$ & $<0.0001$ \\
\hline \multicolumn{5}{|l|}{$\mathrm{CHA}_{2} \mathrm{DS}_{2}$-VASc score } \\
\hline$=0$ & $0(0 \%)$ & $15(2.9 \%)$ & $231(16 \%)$ & $<0.0001$ \\
\hline$=1$ & $3(14.3 \%)$ & $77(15 \%)$ & $384(27 \%)$ & $<0.0001$ \\
\hline$=2$ & $2(9.5 \%)$ & $115(23 \%)$ & $344(24 \%)$ & 0.28 \\
\hline$\geq 3$ & $16(76 \%)$ & $302(59 \%)$ & $473(33 \%)$ & $<0.0001$ \\
\hline HAS-BLED score & $\begin{array}{c}2.8 \pm 1.0 \\
3[2-3] \\
n=18\end{array}$ & $\begin{array}{c}1.8 \pm 1.0 \\
2[1-2] \\
n=407\end{array}$ & $\begin{array}{c}1.1 \pm 0.9 \\
1[0-2] \\
\mathrm{n}=1038\end{array}$ & $<0.0001$ \\
\hline \multicolumn{5}{|l|}{ HAS-BLED score } \\
\hline$=0$ & $0(0 \%)$ & $34(8.4 \%)$ & $336(26 \%)$ & $<0.0001$ \\
\hline$=1$ & $1(5.6 \%)$ & $150(30 \%)$ & $623(44 \%)$ & $<0.0001$ \\
\hline$=2$ & $7(39 \%)$ & $175(34 \%)$ & $404(28 \%)$ & $<0.0001$ \\
\hline$\geq 3$ & $10(56 \%)$ & $146(29 \%)$ & $69(4.8 \%)$ & $<0.0001$ \\
\hline
\end{tabular}


Table 1 (cont.). Clinical characteristics in all groups according to eGFR.

\begin{tabular}{|c|c|c|c|c|}
\hline Variable & $\begin{array}{c}\text { Patients with } \\
\text { eGFR }<30 \\
\text { (n = 21) }\end{array}$ & $\begin{array}{l}\text { Patients with } \\
\text { eGFR 30-59 } \\
\text { (n = 509) }\end{array}$ & $\begin{array}{l}\text { Patients with } \\
\text { eGFR } \geq 60 \\
\text { (n = 1432) }\end{array}$ & $\mathbf{P}$ \\
\hline \multicolumn{5}{|l|}{ Laboratory parameters } \\
\hline Hemoglobin $[\mathrm{g} / \mathrm{dL}]$ & $\begin{array}{c}13[12-14] \\
n=21\end{array}$ & $\begin{array}{c}14[13-15] \\
n=490\end{array}$ & $\begin{array}{c}15 \text { [14-15] } \\
n=1418\end{array}$ & $<0.0001$ \\
\hline Hematocrit [\%] & $\begin{array}{c}39[35-42] \\
n=18\end{array}$ & $\begin{array}{c}42 \text { [39-45]; } \\
\mathrm{n}=391\end{array}$ & $\begin{array}{c}43 \text { [40-46]; } \\
\mathrm{n}=1233\end{array}$ & $<0.0001$ \\
\hline Platelet count $[\mathrm{K} / \mu \mathrm{L}]$ & $\begin{array}{c}175 \text { [156-199]; } \\
n=21\end{array}$ & $\begin{array}{c}217[179-253] \\
n=488\end{array}$ & $\begin{array}{c}216[182-252] \\
\mathrm{n}=1414\end{array}$ & 0.01 \\
\hline Creatinine $[\mathrm{mg}]$ & $2.4[2.0-5.4]$ & $1.3[1.1-1.4]$ & $1.0[0.9-1.1]$ & $<0.0001$ \\
\hline GFR $\left[\mathrm{mL} / \mathrm{min} / 1.73 \mathrm{~m}^{2}\right]$ & 23 [11-28] & 53 [46-57] & 84 [71-90] & $<0.0001$ \\
\hline INR (for patients on VKA) & $\begin{array}{c}2.1[2.0-2.4] \\
n=9\end{array}$ & $\begin{array}{c}2.3[1.8-2.9] \\
n=147\end{array}$ & $\begin{array}{c}2.3[1.8-2.9] \\
\mathrm{n}=461\end{array}$ & 0.63 \\
\hline \multicolumn{5}{|l|}{ INR (for patients on VKA) } \\
\hline$<2.0$ & $4(44 \%)$ & $54(37 \%)$ & $139(30 \%)$ & 0.37 \\
\hline $2.0-3.0$ & $4(44 \%)$ & $61(41 \%)$ & $225(49 \%)$ & 0.35 \\
\hline$>3.0$ & $1(11 \%)$ & $32(22 \%)$ & $97(21 \%)$ & 0.82 \\
\hline APTT [s] & $\begin{array}{c}34[32-48] \\
n=17\end{array}$ & $\begin{array}{c}37 \text { [32-45] } \\
\mathrm{n}=371\end{array}$ & $\begin{array}{c}35[30-42] \\
\mathrm{n}=1171\end{array}$ & $<0.0001$ \\
\hline
\end{tabular}

AF - atrial fibrillation; APTT - activated partial thromboplastin time; BMI - body mass index; CABG — coronary artery bypass graft; $\mathrm{CAD}$ - coronary artery disease; $\mathrm{MI}$ - myocardial infarction; eGFR — estimated glomerular filtration rate; INR — international normalized ratio; $\mathrm{PAD}$ - peripheral artery disease; $\mathrm{PCl}$ - percutaneous coronary intervention; TIA — transient ischemic attack; VKA — vitamin $\mathrm{K}$ antagonists

Table 2. Therapeutic characteristics and echocardiography findings in all groups according to eGFR.

\begin{tabular}{|c|c|c|c|c|}
\hline Variable & $\begin{array}{l}\text { Patients with } \\
\text { eGFR }<30 \\
\text { (n = 21) }\end{array}$ & $\begin{array}{c}\text { Patients with } \\
\text { eGFR 30-59 } \\
\text { (n = 509) }\end{array}$ & $\begin{array}{l}\text { Patients with } \\
\text { eGFR } \geq 60 \\
\text { (n=1432) }\end{array}$ & $\mathbf{P}$ \\
\hline \multicolumn{5}{|l|}{ Type of procedure planned } \\
\hline Cardioversion & $9(43 \%)$ & $246(48 \%)$ & $479(33 \%)$ & $<0.0001$ \\
\hline Ablation & $12(57 \%)$ & $263(52 \%)$ & $953(67 \%)$ & $<0.0001$ \\
\hline \multicolumn{5}{|l|}{ Antithrombotic treatment } \\
\hline No OAC & $2(9.5 \%)$ & $13(2.6 \%)$ & $102(7.1 \%)$ & 0.001 \\
\hline VKA & $9(43 \%)$ & $168(33 \%)$ & $503(35 \%)$ & 0.44 \\
\hline NOAC & $10(48 \%)$ & $328(64 \%)$ & $827(58 \%)$ & 0.01 \\
\hline dabigatran & $5(24 \%)$ & $157(31 \%)$ & $387(27 \%)$ & 0.29 \\
\hline rivaroxaban & $3(14 \%)$ & $160(32 \%)$ & $431(30 \%)$ & 0.18 \\
\hline apixaban & $2(9.5 \%)$ & $11(2.2 \%)$ & $9(0.6 \%)$ & $<0.0001$ \\
\hline Reduced dose of NOAC & $5(24 \%)$ & $53(10 \%)$ & $40(2.8 \%)$ & $<0.0001$ \\
\hline Bridging therapy with heparin & $\begin{array}{c}1(5.6 \%) \\
n=18\end{array}$ & $\begin{array}{c}14(3.4 \%) \\
n=407\end{array}$ & $\begin{array}{c}70(6.7 \%) \\
\mathrm{n}=1031\end{array}$ & 0.05 \\
\hline Antiplatelets & $\begin{array}{c}1(5.6 \%) \\
n=18\end{array}$ & $\begin{array}{c}19(4.7 \%) \\
\mathrm{n}=407\end{array}$ & $\begin{array}{c}62(6.0 \%) \\
\mathrm{n}=1031\end{array}$ & 0.63 \\
\hline \multicolumn{5}{|l|}{ Transthoracic echocardiography* } \\
\hline Ejection fraction [\%] & $\begin{array}{c}50[25-55] \\
n=5\end{array}$ & $\begin{array}{c}55[50-60] \\
n=211\end{array}$ & $\begin{array}{c}58[50-60] \\
n=607\end{array}$ & 0.04 \\
\hline Left atrial diameter $[\mathrm{cm}]$ & $\begin{array}{c}49[48-52] \\
n=6\end{array}$ & $\begin{array}{c}45[42-48] \\
n=216\end{array}$ & $\begin{array}{c}45[41-48] \\
\mathrm{n}=664\end{array}$ & 0.26 \\
\hline \multicolumn{5}{|c|}{ Transesophageal echocardiography* } \\
\hline Thrombus & $5(24 \%)$ & $46(9.0 \%)$ & $57(4.0 \%)$ & $<0.0001$ \\
\hline LAA emptying velocity [cm/s] & $\begin{array}{c}31[25-55] \\
n=7\end{array}$ & $\begin{array}{c}42 \text { [29-64]; } \\
\mathrm{n}=332\end{array}$ & $\begin{array}{c}50[32-74] \\
\mathrm{n}=1130\end{array}$ & $<0.0001$ \\
\hline SEC & $\begin{array}{c}5(25 \%) \\
\mathrm{n}=18\end{array}$ & $\begin{array}{c}97(25 \%) \\
n=392\end{array}$ & $\begin{array}{c}237(19 \%) \\
n=1233\end{array}$ & 0.08 \\
\hline
\end{tabular}

*Performed during index hospitalization. eGFR — estimated glomerular filtration rate; LAA — left atrial appendage; OAC — oral anticoagulants; NOAC — non-vitamin K antagonist oral anticoagulants; SEC — spontaneous echo contrast; VKA — vitamin K antagonists 
Table 3. Logistic regression analyses of predictors of left atrial thrombus in the whole group of patients.

\begin{tabular}{lcccc}
\hline Variable & Univariate analysis & \multicolumn{3}{c}{ Multivariate analysis } \\
\cline { 3 - 5 } & & $\mathbf{O R}$ & $\mathbf{9 5 \%} \mathbf{C l}$ & $\mathbf{P}$ \\
\hline Age & & 1.02 & $1.00-1.05$ & $\mathbf{0 . 0 3}$ \\
Non-paroxysmal AF (vs. paroxysmal AF) & $<\mathbf{0 . 0 0 0 1}$ & 5.62 & $3.10-10.17$ & $<\mathbf{0 . 0 0 0 1}$ \\
Dyslipidemia & $<\mathbf{0 . 0 0 0 1}$ & 0.72 & $0.45-1.15$ & 0.16 \\
Diabetes & $\mathbf{0 . 0 2}$ & 1.40 & $0.87-2.25$ & 0.16 \\
Coronary artery disease & $\mathbf{0 . 0 0 1}$ & 5.47 & $0.40-75.39$ & 0.20 \\
Vascular disease & $\mathbf{0 . 0 2}$ & 0.14 & $0.01-1.93$ & 0.14 \\
Myocardial infraction & $\mathbf{0 . 0 4 5}$ & 1.09 & $0.48-2.50$ & 0.83 \\
Heart failure & $\mathbf{0 . 0 3}$ & 2.22 & $1.42-3.47$ & $<\mathbf{0 . 0 0 0 1}$ \\
Previous bleeding & $<\mathbf{0 . 0 0 0 1}$ & $\mathbf{0 . 0 0}$ & $\mathbf{0 . 0 0 1}$ \\
eGFR & $<\mathbf{0 . 0 0 0 1}$ & $\mathbf{0 . 9 7}$ & $1.56-5.65$ & $\mathbf{0 . 0 4}$ \\
\hline
\end{tabular}

$\mathrm{AF}$ — atrial fibrillation; $\mathrm{Cl}$ — confidence intervals; eGFR — estimated glomerular filtration rate; OR — odds ratio

Table 4. Logistic regression analyses of predictors of left atrial thrombus.

\begin{tabular}{|c|c|c|c|c|}
\hline \multicolumn{5}{|c|}{ A. Predictors of left atrial thrombus in the group of patients with eGFR $60 \mathrm{~mL} / \mathrm{min} / 1.73 \mathrm{~m}^{2}$ or more } \\
\hline \multirow[t]{2}{*}{ Variable } & \multirow[t]{2}{*}{ Univariate analysis } & \multicolumn{3}{|c|}{ Multivariate analysis } \\
\hline & & OR & $95 \% \mathrm{Cl}$ & $\mathbf{P}$ \\
\hline Age & 0.001 & 1.03 & $1.00-1.06$ & 0.047 \\
\hline Non-paroxysmal AF (vs paroxysmal AF) & $<0.0001$ & 4.49 & $2.12-9.48$ & $<0.0001$ \\
\hline Diabetes & $<0.0001$ & 1.66 & $0.90-3.04$ & 0.10 \\
\hline Heart failure & $<0.0001$ & 2.35 & $1.31-4.23$ & 0.004 \\
\hline Previous bleeding & 0.002 & 3.64 & $1.49-8.92$ & 0.005 \\
\hline \multicolumn{5}{|c|}{ B. Predictors of left atrial thrombus in the group of patients with eGFR of less than $60 \mathrm{~mL} / \mathrm{min} / 1.73 \mathrm{~m}{ }^{2}$} \\
\hline \multirow[t]{2}{*}{ Variable } & \multirow[t]{2}{*}{ Univariate analysis } & \multicolumn{3}{|c|}{ Multivariate analysis } \\
\hline & & OR & $95 \% \mathrm{Cl}$ & $\mathbf{P}$ \\
\hline Non-paroxysmal AF (vs. paroxysmal AF) & $<0.0001$ & 6.72 & $2.49-18.17$ & $<0.0001$ \\
\hline Hypertension & 0.048 & 0.46 & $0.23-0.91$ & 0.03 \\
\hline Dyslipidemia & 0.04 & 0.65 & $0.31-1.36$ & 0.25 \\
\hline Heart failure & 0.002 & 2.25 & $1.18-4.27$ & 0.01 \\
\hline Previous bleeding & 0.02 & 2.84 & $1.16-6.99$ & 0.02 \\
\hline
\end{tabular}

$\mathrm{AF}$ - atrial fibrillation; $\mathrm{Cl}$ - confidence intervals; OR — odds ratio; eGFR — estimated glomerular filtration rate

point of stroke or systemic embolism occurred in 1.57 per 100 patient-years with rivaroxaban $20 \mathrm{mg} /$ /day vs. 2.00 per 100 patient-years with warfarin [18]. The ARISTOTLE study shows that apixaban at doses of $5 \mathrm{mg}$ twice daily in eGFR categories $>80,>50$ to $80, \leq 50 \mathrm{~mL} / \mathrm{min} / 1.73 \mathrm{~m}^{2}$ was generally superior in preventing thromboembolic events with no significant interaction between the treatment effect [19]. According to the RE-LY study, the annual rates of thromboembolic events among patient eGFR categories $>80,50$ to $<80,<50 \mathrm{~mL} /$
$/ \mathrm{min} / 1.73 \mathrm{~m}^{2}$ were lower with dabigatran $150 \mathrm{mg}$ and similar with $110 \mathrm{mg}$ twice daily compared with warfarin without significant heterogeneity in subgroups defined by renal function (interaction [20]. However, another sub-analysis of the RE-LY trial data showed a significantly faster rate of decline in renal function in patients on VKA compared with those on dabigatran [21]. Using data from the IMS Disease Analyzer Germany study, Posch et al. [22] proved that exposure to VKA is associated with accelerated eGFR decline. In 7409 patients 


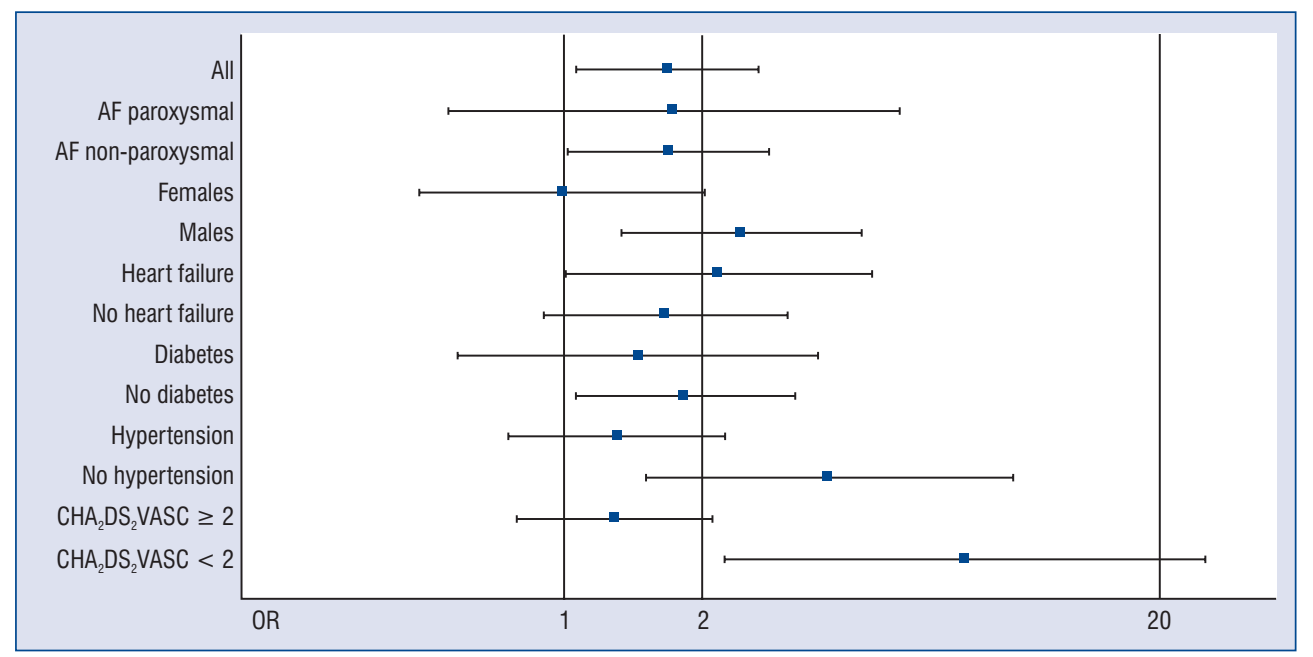

Figure 1. Forest plot of chronic kidney disease as predictor of left atrial appendage thrombus in atrial fibrillation patients depending on additional risk factors; AF — atrial fibrillation; OR — odds ratio.

Table 5. The distribution of anticoagulation treatment in patients with left atrial appendage (LAA) thrombus glomerular filtration rate less than $60 \mathrm{~mL} / \mathrm{min} / 1.73 \mathrm{~m}^{2}$ and $60 \mathrm{~mL} / \mathrm{min} / 1.73 \mathrm{~m}^{2}$ and more.

\begin{tabular}{lccc}
\hline Variable & \multicolumn{3}{c}{ Patients with LAA thrombus } \\
\cline { 2 - 4 } & GFR $<\mathbf{6 0}(\mathbf{n}=\mathbf{5 1})$ & GFR $\geq \mathbf{6 0}(\mathbf{n}=\mathbf{5 7})$ & $\mathbf{P}$ \\
\hline No OAC & $3(5.9 \%)$ & $3(5.3 \%)$ & 1.00 \\
VKA & $23(45 \%)$ & $28(49 \%)$ & 0.85 \\
NOAC & $25(49 \%)$ & $26(46 \%)$ & 0.85 \\
dabigatran & $14(46 \%)$ & $14(25 \%)$ & 0.52 \\
rivaroxaban & $12(21 \%)$ & $12(21 \%)$ & 0.64 \\
apixaban & $0(0 \%)$ & $0(0 \%)$ & 1.00 \\
\hline
\end{tabular}

NOAC — non-vitamin K antagonist oral anticoagulants; OAC — oral anticoagulants; VKA — vitamin K antagonists

Table 6. The distribution of left atrial appendage (LAA) thrombus in patients with reduced non-vitamin $\mathrm{K}$ antagonist oral anticoagulants (NOAC) according to glomerular filtration rate (GFR).

\begin{tabular}{lcccc}
\hline Variable & \multicolumn{4}{c}{ Patients with reduced NOAC } \\
\cline { 2 - 5 } & GFR $<30(\mathrm{n}=5)$ & GFR $30-59(\mathrm{n}=53)$ & GFR $\geq \mathbf{6 0}(\mathbf{n}=\mathbf{4 0})$ & $\mathrm{P}$ \\
\hline LAA thrombus & $1(20 \%)$ & $5(9.4 \%)$ & $1(2.5 \%)$ & 0.001 \\
\hline
\end{tabular}

with VKA exposure, real failure progression was significantly faster compared to patients without VKA exposure (5-year absolute eGFR loss from baseline: 6.0 vs. $4.5 \mathrm{~mL} / \mathrm{min} / 1.73 \mathrm{~m}^{2}$ ) [22]. It has been suggested that VKA may lead to decreased renal function via repeated subclinical glomerular hemorrhages or through accelerated tissue or vascular calcification [23].
There is no conclusive research that has determined the superiority of one of the NOAC drugs. Noteworthy, all NOAC are at least partly eliminated by the kidneys. In contrast to dabigatran $(80 \%)$ and rivaroxaban $(35 \%)$, apixaban is less dependent on renal elimination (27\%) and is labeled for use in end-stage kidney disease. It may explain results from a Falissard et al. [24] study, in 
Table 7. Comparison of patients without oral anticoagulation according to glomerular filtration rate.

\begin{tabular}{|c|c|c|c|}
\hline \multirow[t]{2}{*}{ Variable } & \multicolumn{3}{|c|}{ No OAC } \\
\hline & GFR $<60(n=15)$ & GFR $\geq 60(n=102)$ & $\mathbf{P}$ \\
\hline Age [years] & $63[58-71]$ & $53[38-60]$ & 0.007 \\
\hline Female & $7(47 \%)$ & $28(28 \%)$ & 0.14 \\
\hline BMI $\left[\mathrm{kg} / \mathrm{m}^{2}\right]$ & $29[27-31] ; n=12$ & $27[25-31] ; n=97$ & 0.39 \\
\hline Obesity & $4(27 \%)$ & $31(30 \%)$ & 1.00 \\
\hline \multicolumn{4}{|l|}{ Type of AF } \\
\hline Paroxysmal AF & $8(53 \%)$ & $89(87 \%)$ & 0.004 \\
\hline Persistent AF & $5(33 \%)$ & $11(11 \%)$ & 0.03 \\
\hline Permanent/long-standing persistent AF & $2(13 \%)$ & $2(2.0 \%)$ & 0.08 \\
\hline \multicolumn{4}{|l|}{ Type of procedure planned } \\
\hline Cardioversion & $6(40 \%)$ & $17(17 \%)$ & 0.07 \\
\hline Ablation & $9(60 \%)$ & $85(83 \%)$ & 0.07 \\
\hline \multicolumn{4}{|l|}{ Concomitant diseases } \\
\hline Hypertension & $8(53 \%)$ & $40(39 \%)$ & 0.40 \\
\hline Dyslipidemia & $1(6.7 \%)$ & $31(30 \%)$ & 0.07 \\
\hline Diabetes & $2(13 \%)$ & $3(2.9 \%)$ & 0.12 \\
\hline CAD & $3(20 \%)$ & $8(7.8 \%)$ & 0.15 \\
\hline Previous myocardial infarction & $2(13 \%)$ & $6(5.9 \%)$ & 0.27 \\
\hline Previous $\mathrm{CABG} / \mathrm{PCl}$ & $2(13 \%)$ & $5(4.9 \%)$ & 0.22 \\
\hline PAD & $2(13 \%)$ & $1(1.0 \%)$ & 0.04 \\
\hline Vascular disease (CAD and/or PAD) & $3(20 \%)$ & $9(8.8 \%)$ & 0.18 \\
\hline Heart failure & $5(33 \%)$ & $10(9.8 \%)$ & 0.02 \\
\hline Previous ischemic stroke/TIA & $2(13 \%)$ & $2(2.0 \%)$ & 0.08 \\
\hline Previous ischemic stroke/TIA/peripheral embolism & $2(13 \%)$ & $2(2.0 \%)$ & 0.08 \\
\hline Chronic respiratory disease & $1(6.7 \%)$ & $2(2.0 \%)$ & 0.34 \\
\hline Liver disease & $0(0 \%)$ & $0(0 \%)$ & 1.00 \\
\hline Hyperthyroidism & $1(6.7 \%)$ & $3(2.9 \%)$ & 0.43 \\
\hline Hypothyroidism & $3(20 \%)$ & $8(7.8 \%)$ & 0.15 \\
\hline Malignancy & $0(0 \%)$ & $1(1.0 \%)$ & 1.00 \\
\hline Previous bleeding & $4(27 \%)$ & $4(3.9 \%)$ & 0.009 \\
\hline Smoking & $5(33 \%)$ & $34(33 \%)$ & 1.00 \\
\hline \multicolumn{4}{|l|}{ Thromboembolic risk } \\
\hline $\mathrm{CHADS}_{2}$ score & $1[0.5-2]$ & $0[0-1]$ & 0.007 \\
\hline $\mathrm{CHA}_{2} \mathrm{DS}_{2}$-VASc score & $2[1-3]$ & $1[0-2]$ & 0.01 \\
\hline \multicolumn{4}{|l|}{$\mathrm{CHA}_{2} \mathrm{DS}_{2}$-VASc score } \\
\hline$=0$ & $0(0 \%)$ & $24(24 \%)$ & 0.04 \\
\hline$=1$ & $6(40 \%)$ & $23(23 \%)$ & 0.20 \\
\hline$=2$ & $4(27 \%)$ & $25(25 \%)$ & 1.00 \\
\hline$\geq 3$ & $5(33 \%)$ & $30(29 \%)$ & 0.77 \\
\hline HAS-BLED score & $1[1-3]$ & $1[0-1.8]$ & 0.02 \\
\hline \multicolumn{4}{|l|}{ HAS-BLED score } \\
\hline$=0$ & $2(13 \%)$ & $27(27 \%)$ & 0.35 \\
\hline$=1$ & $3(20 \%)$ & $36(35 \%)$ & 0.38 \\
\hline$=2$ & $7(47 \%)$ & $24(24 \%)$ & 0.07 \\
\hline$\geq 3$ & $3(20 \%)$ & $15(15 \%)$ & 0.70 \\
\hline
\end{tabular}


Table 7 (cont.). Comparison of patients without oral anticoagulation according to glomerular filtration rate.

\begin{tabular}{|c|c|c|c|}
\hline \multirow[t]{2}{*}{ Variable } & \multicolumn{3}{|c|}{ No OAC } \\
\hline & GFR $<60(n=15)$ & GFR $\geq 60$ (n = 102) & $\mathbf{P}$ \\
\hline \multicolumn{4}{|l|}{ Laboratory parameters } \\
\hline Hemoglobin $[\mathrm{g} / \mathrm{dL}]$ & 13 [12-15] & 15 [14-15] & 0.005 \\
\hline Hematocrit [\%] & 41 [38-43] & $44[42-46]$ & 0.003 \\
\hline WBC $[\mathrm{K} / \mu \mathrm{L}]$ & $7.4[6.6-8.6]$ & $7.1[6.1-8.8]$ & 0.73 \\
\hline Platelet count $[\mathrm{K} / \mu \mathrm{L}]$ & 254 [196-284] & 228 [199-256] & 0.41 \\
\hline AST & $25[20-39] ; n=14$ & $22[19-27] ; n=93$ & 0.27 \\
\hline ALT & $31[21-46] ; n=14$ & $32[23-38] ; n=94$ & 0.88 \\
\hline \multicolumn{4}{|c|}{ Transthoracic echocardiography* } \\
\hline Ejection fraction [\%] & $50 \pm 0 ; n=1$ & $60[55-62] ; n=17$ & 0.29 \\
\hline Left atrial diameter $[\mathrm{cm}]$ & $44[43-47] ; n=4$ & $43[40-45] ; n=44$ & 0.71 \\
\hline \multicolumn{4}{|c|}{ Transesophageal echocardiography* } \\
\hline Thrombus & $3(20 \%)$ & $3(2.9 \%)$ & 0.03 \\
\hline LAA emptying velocity $[\mathrm{cm} / \mathrm{s}]$ & $56[42-68] ; n=15$ & 70 [49-87]; $\mathrm{n}=99$ & 0.04 \\
\hline SEC & $2(13 \%)$ & $7(6.9 \%)$ & 0.32 \\
\hline
\end{tabular}

*Performed during index hospitalization. AST — aspartate transaminase; AF - atrial fibrillation; ALT — alanine transaminase; BMI — body mass index; CABG — coronary artery bypass grafting; CAD — coronary artery disease; GFR — glomerular filtration rate; LAA — left atrial appendage; OAC - oral anticoagulants; $\mathrm{PAD}$ - peripheral artery disease; $\mathrm{PCl}$ - percutaneous coronary intervention; SEC — spontaneous echo contrast; TIA — transient ischemic attack; WBC — white blood cells

which apixaban was more likely to be prescribed than other NOACs in patients with decreased renal function. Based on online an survey created to analyze the opinion of the role of OAC in various clinical settings, edoxaban and apixaban were the favorites for patients with $\mathrm{AF}$ and moderate CKD [25]. In the present study, apixaban was prescribed more often in patients with kidney failure than in patients with normal kidney function. However, the prevalence of apixaban therapy was the lowest among all NOACs.

Recent publications have demonstrated the limitation of the $\mathrm{CHA}_{2} \mathrm{DS}_{2}$-VASc score for predicting future strokes in patients with $\mathrm{AF}$ [26]. There are conflicting data as to whether the integration of renal function parameters into $\mathrm{CHA}_{2} \mathrm{DS}_{2}$-VASc score could improve its predictive value. Some of studies suggest that the predictive value of $\mathrm{CHA}_{2} \mathrm{DS}_{2}$-VASc score is not improved by the addition of renal status because the factors within $\mathrm{CHA}_{2} \mathrm{DS}_{2}$-VASc are themselves related to renal dysfunction [27]. On the other hand, previous results from Global Anticoagulant Registry in the FIELD-Atrial Fibrillation (GARFIELD-AF) registry shows that moderate/severe CKD is independently associated with a higher risk of stroke/systemic embolism [28]. These findings are consistent with a previous study evaluating new thromboembolic risk score i.e. $\mathrm{CHA}_{2} \mathrm{DS}_{2}$-VASc-
RAF score included two additional parameters i.e. renal dysfunction and $\mathrm{AF}$ type. Both variables proved strong, independent predictors of LAA thrombus on TEE and improve thromboembolic risk stratification [29]. Moreover, an inverse correlation between eGFR values and LAA thrombus occurrence were observed among patients included in the current study $(24 \%, 9 \%$ and $4 \%$ of patients with eGFR $<30,30-59$ and $\geq 60 \mathrm{~mL} / \mathrm{min} / 1.73 \mathrm{~m}^{2}$, respectively). This is in line with study by Kizawa et al. [30], that examined $581 \mathrm{AF}$ patients with CKD stages 1-4. The prevalence of thrombogenic milieu (LA thrombus, dense SEC, or LAA a velocity $\leq 25 \mathrm{~cm} / \mathrm{s}$ ) increased with decreasing eGFR $(4 \%, 18 \%, 36 \%$, and $86 \%$ for each group, $\mathrm{p}<$ $<0.001)$. Moreover, multivariate logistic regression analysis revealed that every $10 \mathrm{~mL} / \mathrm{min} / 1.73 \mathrm{~m}^{2}$ decrement in eGFR was a significant independent correlate of thrombogenic milieu (odds ratio 0.80 , $\mathrm{p}=0.005)$ [30].

It is unclear, whether patients with decreased renal function and $\mathrm{AF}$ benefit from $\mathrm{OAC}$ to the same extent as those with normal kidney function. Current evidence suggests that patients with $\mathrm{AF}$ who have CKD with eGFR $>15 \mathrm{~mL} / \mathrm{min} / 1.73 \mathrm{~m}^{2}$ should be treated with OAC if they have an at least an intermediate risk of embolization, as assessed with the $\mathrm{CHA}_{2} \mathrm{DS}_{2}$-VASc score [31]. In the present study, $98 \%$ of patients with decreased renal func- 
tion treated with OAC were at moderate or high risk $\left(\mathrm{CHA}_{2} \mathrm{DS}_{2}-\mathrm{VASc}\right.$ score $\left.\geq 2\right)$. Among high risk patients in whom OAC are recommended, thrombus was more frequent in patients with lower eGFR. Moreover, among patients who were not treated with OAC LAA thrombus occurred more often in patients with eGFR $<60 \mathrm{~mL} / \mathrm{min} / 1.73 \mathrm{~m}^{2}$.

Independent predictors for LAA thrombus formation included the following clinical risk factors - non-paroxysmal AF, hypertension, heart failure, previous bleeding in patients with eGFR $<60 \mathrm{~mL} / \mathrm{min} / 1.73 \mathrm{~m}^{2}$, and age, non-paroxysmal $\mathrm{AF}$, heart failure, previous bleeding in those with eGFR $\geq 60 \mathrm{~mL} / \mathrm{min} / 1.73 \mathrm{~m}^{2}$. This is consistent with the high risk associated with such comorbidities in AF patients [32-34].

Based on previous meta-analysis by Wang et al. [35], patients eligible for a reduced dose of NOAC are at elevated risk of thromboembolic complications when compared to those eligible for full dose of NOAC (2.70\% vs. $4.35 \%$, respectively). In the current study it was confirmed that patients with reduced NOAC a prevalence of LAA thrombus is higher in patients with lower eGFR.

Therefore, there is a particular need to use adequate OAC treatment in patients with CKD.

\section{Conclusions}

The incidence of LAA thrombi was higher in patients with lower eGFR. eGFR was one of the predictors of LAA thrombus. CKD was the predictor of LAA thrombus in all patients as well as in patients with non-paroxysmal AF, in males, without diabetes, without hypertension and with $\mathrm{CHA}_{2} \mathrm{DS}_{2}$-VASc $<2$ that is in groups which are not included in classic risk factors.

\section{Acknowledgments}

The authors thank Paweł Piłkowski for his assistance in the statistical analysis, and students: Aldona Babiarz, Aleksandra Bodys, Robert Uliński, and Maciej Żochowski for their assistance in data collection.

Conflict of interest: Iwona Gorczyca - Honoraria for lectures from Bayer, Boehringer Ingelheim; Beata Wożakowska-Kapłon - Honoraria for lectures from Bayer, Boehringer Ingelheim, Pfeizer; Krzysztof J. Filipiak - Honoraria for lectures from Bayer, Boehringer Ingelheim, MSD, Pfeizer; Grzegorz Opolski - Honoraria for lectures from Bayer, Boehringer Ingelheim, Pfeizer; Agnieszka Kapłon-Cieślicka - Honoraria for lectures/travel grants from Bayer, Boehringer Ingelheim, MSD, Pfeizer.

\section{References}

1. Chugh SS, Havmoeller R, Narayanan K, et al. Worldwide epidemiology of atrial fibrillation: a Global Burden of Disease 2010 Study. Circulation. 2014; 129(8): 837-847, doi: 10.1161/CIRCULATIONAHA.113.005119, indexed in Pubmed: 24345399.

2. Haim M, Hoshen M, Reges O, et al. Prospective national study of the prevalence, incidence, management and outcome of a large contemporary cohort of patients with incident non-valvular atrial fibrillation. J Am Heart Assoc. 2015; 4(1): e001486, doi: 10.1161/ JAHA.114.001486, indexed in Pubmed: 25609415.

3. Kannel WB, Wolf PA, Benjamin EJ, et al. Prevalence, incidence, prognosis, and predisposing conditions for atrial fibrillation: population-based estimates. Am J Cardiol. 1998; 82(8A): 2N-9N, doi: 10.1016/s0002-9149(98)00583-9, indexed in Pubmed: 9809895.

4. Gorczyca I, Michalska A, Chrapek M, et al. Non-vitamin K antagonist oral anticoagulants in patients with atrial fibrillation in secondary stroke and systemic embolism prevention. Cardiol J. 2019 [Epub ahead of print], doi: 10.5603/CJ.a2019.0069, indexed in Pubmed: 31313276.

5. Ruff CT, Giugliano RP, Braunwald E, et al. Comparison of the efficacy and safety of new oral anticoagulants with warfarin in patients with atrial fibrillation: a meta-analysis of randomised trials. Lancet. 2014; 383(9921): 955-962, doi: 10.1016/S01406736(13)62343-0, indexed in Pubmed: 24315724.

6. Olesen J, Lip G, Kamper AL, et al. Stroke and bleeding in atrial fibrillation with chronic kidney disease. N Engl J Med. 2012; 367(7): 625-635, doi: 10.1056/nejmoa1105594.

7. Albertsen I, Rasmussen L, Overvad T, et al. Risk of stroke or systemic embolism in atrial fibrillation patients treated with warfarin. Stroke. 2013; 44(5): 1329-1336, doi: 10.1161/strokeaha.113.000883.

8. Connolly S, Ezekowitz M, Yusuf S, et al. Dabigatran versus warfarin in patients with atrial fibrillation. N Engl J Med. 2009; 361(12): 1139-1151, doi: 10.1056/nejmoa0905561.

9. Granger CB, Alexander JH, McMurray JJV, et al. ARISTOTLE Committees and Investigators. Apixaban versus warfarin in patients with atrial fibrillation. N Engl J Med. 2011; 365(11): 981-992, doi: 10.1056/NEJMoa1107039, indexed in Pubmed: 21870978.

10. Patel M, Mahaffey K, Garg J, et al. Rivaroxaban versus Warfarin in Nonvalvular Atrial Fibrillation. N Engl J Med. 2011; 365(10): 883-891, doi: 10.1056/nejmoa1009638.

11. Giugliano RP, Ruff CT, Braunwald E, et al. ENGAGE AF-TIMI 48 Investigators. Edoxaban versus warfarin in patients with atrial fibrillation. N Engl J Med. 2013; 369(22): 2093-2104, doi: 10.1056/ NEJMoa1310907, indexed in Pubmed: 24251359.

12. Heidbuchel H, Verhamme P, Alings M, et al. ESC Scientific Document Group. Updated European Heart Rhythm Association Practical Guide on the use of non-vitamin K antagonist anticoagulants in patients with non-valvular atrial fibrillation. Europace. 2015; 17(10): 1467-1507, doi: 10.1093/europace/euv309, indexed in Pubmed: 26324838.

13. Kosmalska K, Rzyman M, Miękus P, et al. Usefulness of transesophageal echocardiography before cardioversion in atrial arrhythmias. Cardiol J. 2019 [Epub ahead of print], doi: 10.5603/ CJ.a2019.0056, indexed in Pubmed: 31225630.

14. Gawałko M, Kapłon-Cieślicka A, Budnik M, et al. Comparison of different oral anticoagulant regimens in patients with atrial fibrillation undergoing ablation or cardioversion. Pol Arch Intern 
Med. 2017; 127(12): 823-831, doi: 10.20452/pamw.4117, indexed in Pubmed: 28972957.

15. Loo SY, Coulombe J, Dell'Aniello S, et al. Comparative effectiveness of novel oral anticoagulants in UK patients with nonvalvular atrial fibrillation and chronic kidney disease: a matched cohort study. BMJ Open. 2018; 8(1): e019638, doi: 10.1136/bmjopen-2017-019638, indexed in Pubmed: 29371284.

16. Kimachi M, Furukawa TA, Kimachi K, et al. Direct oral anticoagulants versus warfarin for preventing stroke and systemic embolic events among atrial fibrillation patients with chronic kidney disease. Cochrane Database Syst Rev. 2017; 11: CD011373, doi: 10.1002/14651858.CD011373.pub2, indexed in Pubmed: 29105079.

17. Shin JI, Secora A, Alexander GC, et al. Risks and Benefits of Direct Oral Anticoagulants across the Spectrum of GFR among Incident and Prevalent Patients with Atrial Fibrillation. Clin J Am Soc Nephrol. 2018; 13(8): 1144-1152, doi: 10.2215/ CJN.13811217, indexed in Pubmed: 30002224.

18. Fox KAA, Piccini JP, Wojdyla D, et al. Prevention of stroke and systemic embolism with rivaroxaban compared with warfarin in patients with non-valvular atrial fibrillation and moderate renal impairment. Eur Heart J. 2011; 32(19): 2387-2394, doi: 10.1093/ eurheartj/ehr342, indexed in Pubmed: 21873708.

19. Hohnloser SH, Hijazi Z, Thomas L, et al. Efficacy of apixaban when compared with warfarin in relation to renal function in patients with atrial fibrillation: insights from the ARISTOTLE trial. Eur Heart J. 2012; 33(22): 2821-2830, doi: 10.1093/eurheartj/ ehs274, indexed in Pubmed: 22933567.

20. Hijazi Z, Hohnloser SH, Oldgren J, et al. Efficacy and safety of dabigatran compared with warfarin in relation to baseline renal function in patients with atrial fibrillation: a RE-LY (Randomized Evaluation of Long-term Anticoagulation Therapy) trial analysis. Circulation. 2014; 129(9): 961-970, doi: 10.1161/CIRCULATIONAHA.113.003628, indexed in Pubmed: 24323795.

21. Böhm M, Ezekowitz MD, Connolly SJ, et al. Changes in renal function in patients with atrial fibrillation: an analysis from the RE-LY trial. J Am Coll Cardiol. 2015; 65(23): 2481-2493, doi: 10.1016/j.jacc.2015.03.577, indexed in Pubmed: 26065986.

22. Posch F, Ay C, Stöger H, et al. Exposure to vitamin k antagonists and kidney function decline in patients with atrial fibrillation and chronic kidney disease. Res Pract Thromb Haemost. 2019; 3(2): 207-216, doi: 10.1002/rth2.12189, indexed in Pubmed: 31011705.

23. Zaragatski E, Grommes J, Schurgers LJ, et al. Vitamin K antagonism aggravates chronic kidney disease-induced neointimal hyperplasia and calcification in arterialized veins: role of vitamin K treatment? Kidney Int. 2016; 89(3): 601-611, doi: 10.1038/ ki.2015.298, indexed in Pubmed: 26466318.

24. Falissard B, Picard F, Mahe I, et al. Apixaban for prevention of stroke and systemic embolism in patients with non-valvular atrial fibrillation in France: The PAROS cross-sectional study of routine clinical practice. Arch Cardiovasc Dis. 2019; 112(6-7): 400-409, doi: 10.1016/j.acvd.2019.02.003, indexed in Pubmed: 31014991.

25. Chueca Fernández E, López Granados A, Zuazola Martínez MD, et al. Consensus in cardiology on non-vitamin-K oral anticoagulants for patients with atrial fibrillation. Curr Med Res Opin. 2019; 35(9): 1571-1582, doi: 10.1080/03007995.2019.1605049, indexed in Pubmed: 30957564.

26. Quinn GR, Severdija ON, Chang Y, et al. Wide variation in reported rates of stroke across cohorts of patients with atrial fibrillation. Circulation. 2017; 135(3): 208-219, doi: 10.1161/CIRCULATIONAHA.116.024057, indexed in Pubmed: 27799272.

27. Roldán V, Marín F, Manzano-Fernandez S, et al. Does chronic kidney disease improve the predictive value of the CHADS2 and CHA2DS2-VASc stroke stratification risk scores for atrial fibrillation? Thromb Haemost. 2013; 109(5): 956-960, doi: 10.1160/ TH13-01-0054, indexed in Pubmed: 23572113.

28. Goto S, Angchaisuksiri P, Bassand JP, et al. GARFIELD-AF Investigators. Management and 1-Year Outcomes of Patients With Newly Diagnosed Atrial Fibrillation and Chronic Kidney Disease: Results From the Prospective GARFIELD - AF Registry. J Am Heart Assoc. 2019; 8(3): e010510, doi: 10.1161/ JAHA.118.010510, indexed in Pubmed: 30717616.

29. Kapłon-Cieślicka A, Budnik M, Gawałko M, et al. Atrial fibrillation type and renal dysfunction as important predictors of left atrial thrombus. Heart. 2019; 105(17): 1310-1315, doi: 10.1136/ heartjnl-2018-314492, indexed in Pubmed: 31040170.

30. Kizawa S, Ito T, Akamatsu K, et al. Chronic kidney disease as a possible predictor of left atrial thrombogenic milieu among patients with nonvalvular atrial fibrillation. Am J Cardiol. 2018; 122(12): 2062-2067, doi: 10.1016/j.amjcard.2018.08.058, indexed in Pubmed: 30293657.

31. Heine GH, Brandenburg V, Schirmer SH. Oral anticoagulation in chronic kidney disease and atrial fibrillation. Dtsch Arztebl Int. 2018; 115: 287-294.

32. Pang H, Han B, Fu Q, et al. Severity of hypertension correlates with risk of thromboembolic stroke. J Cardiovasc Transl Res. 2017; 10(4): 368-373, doi: 10.1007/s12265-017-9754-0, indexed in Pubmed: 28567670.

33. Olesen J, Lip G, Kamper AL, et al. Stroke and bleeding in atrial fibrillation with chronic kidney disease. N Engl J Med. 2012; 367(7): 625-635, doi: 10.1056/nejmoa1105594.

34. Lau YC, Lane DA, Lip GYH. Atrial fibrillation and heart failure: a bad combination. Am J Cardiol. 2014; 113(7): 1196-1197, doi: 10.1016/j.amjcard.2014.01.002, indexed in Pubmed: 24530002.

35. Wang KL, Lopes RD, Patel MR, et al. Efficacy and safety of reduced-dose non-vitamin K antagonist oral anticoagulants in patients with atrial fibrillation: a meta-analysis of randomized controlled trials. Eur Heart J. 2019; 40(19): 1492-1500, doi: 10.1093/ eurheartj/ehy802, indexed in Pubmed: 30590440. 